\title{
Aspectos do parasitismo de Plasmodium (Novyella) juxtanucleare Versiani \& Gomes, 1941 em Gallus gallus L., 1758 em criação rústica no município de Santa Bárbara do Tugúrio - MG
}

\author{
Aspects of the parasitism of Plasmodium (Novyella) juxtanucleare \\ Versiani \& Gomes, 1941 in Gallus gallus L. 1758, in a rustic raising in \\ Santa Bárbara do Tugúrio - MG
}

\author{
Helba Helena Santos Prezoto, * Michelle Carvalho Maia, ${ }^{\star}$ Isabel Cristina Vidal Siqueira, ${ }^{*}$ Marta D’Agosto**
}

\begin{abstract}
Resumo
Com o objetivo de se ampliar o conhecimento sobre a ocorrência do parasitismo por Plasmodium (Novyella) juxtanucleare Versiani e Gomes, 1941, em aves da espécie Gallus gallus L. 1758 no estado de Minas Gerais, realizou-se um levantamento de prevalência no município de Santa Bárbara do Tugúrio (2115'12"S, 4327'16"W). Foram examinados esfregaços sangüíneos, corados pelo Giemsa e observados em microscopia fotônica em imersão, de 30 aves mestiças, adultas, de criação rústica, mantidas extensivamente, com peso médio de $2,18 \mathrm{~kg} \pm 0,34$, sendo 29 fêmeas. As formas eritrocitárias foram registradas e quantificadas pela observação de 50 campos microscópicos assinalando-se o número de eritrócitos parasitados. A prevalência foi de $100 \%$ e a intensidade média de 7,93 parasitos por lâmina nos campos examinados. Observaram-se em $33,3 \%$ dos esfregaços de um a cinco parasitos, $40 \%$ de seis a 10 e $26,6 \%$ acima de 10 . Não foram observados casos de poliparasitismo.
\end{abstract}

Palavras-chave: Plasmodium juxtanucleare, Gallus gallus, malária aviária, criação rústica.

\begin{abstract}
With the aim of amplity the knowledge about the occurrence of parasitism by Plasmodium (Novyella) juxtanucleare Versiani e Gomes, 1941 in fowls from the species Gallus gallus L., 1758 in the State of Minas Gerais, a search for prevalence was held in Santa Bárbara do Tugúrio (211'12"S, 4327'16”W). We have examined bloody platinum loop for smears, which were dyed through Giemsa and watched in photonic microscopy in immersion, of 30 half-breed birds, which were adults, from rustic raising, kept extensively, with an average weigh of $2,18 \mathrm{Kg} \pm 0,34$, from which 29 were females. The erythocyte forms were registered and quantified through the observation of 50 microscopically fields, and the riumber of parasitized erythrocytes was marked. The prevalence was of $100 \%$ and average intensity of 7,93 parasites per glass in the examined fields. We have observated from one to five parasites in $33,3 \%$ of the platinum loop for smears, from six to ten in $40 \%$ and from ten on in $26,6 \%$. We have not observated any case of poliparasitism.
\end{abstract}

Keywords: Plasmodium juxtanucleare, Gallus gallus, avian malaria, and rustic raising.

\section{Introdução}

Os parasitos causadores da malária aviária foram alguns dos primeiros plasmódios estudados, sendo encontrados em todos os continentes e provavelmente em todos os países, em torno de 450 espécies de aves migratórias ou não (Garnham, 1966). No Brasil, registrou-se Plasmodium juxtanucleare (Versiani e Gomes, 1941), nos estados de Minas Gerais (Versiani e Gomes, 1941, 1943; Paraense, 1949, Krettli, 1971), Rio de Janeiro (Massard, 1976,1979,1982; Sou- za, 1998; Soares et al., 1999), Espírito Santo (Massard, 1976), Pará (Serra Freire e Massard, 1976), Mato Grosso do Sul (Serra Freire e Massard, 1979) e Pernambuco (Mota, 1997).

Várias são as espécies do gênero Plasmodium que acometem as aves. A malária aviária causada por $P$. juxtanucleare em Gallus gallus L., 1758, é a única plasmodiose que ocorre em galináceos no Brasil. A denominação de $P$. juxtanucleare deve-se ao fato da freqüente justaposição deste em relação ao núcleo das células parasitadas. As formas sangüíneas

\footnotetext{
* Mestres em Ciências Biológicas (Zoologia) - Comportamento e Ecologia Animal, ICB, UFJF. Universidade Federal de Juiz de Fora, MG. helba.santos@bol.com.br, michelle.maia@bol.com.br, siqueiraicv@bol.com.br.

** Departamento de Zoologia, ICB, Universidade Federal de Juiz de Fora, Juiz de Fora, 36036-330, MG. dagosto @icb.ufj.br
} 
são de pequenas dimensões e apresentam alta especificidade para G. gallus (Versiani e Gomes, 1941; 1943).

Descrita em aves do estado de Minas Gerais, causa doença crônica acarretando, de acordo com diversos autores, altas taxas de morbidade e mortalidade nos seus hospedeiros vertebrados, podendo causar queda de produção em aves de criação industrial.

Sobre a sua biologia, $P$. juxtanucleare pode ser encontrado nas formas eritrocíticas e exoeritrocíticas. Na primeira é encontrado freqüentemente justaposto ao núcleo do eritrócito. $\mathrm{Na}$ segunda, diversos autores relatam sua presença em órgãos como baço, cérebro, pulmão, medula óssea, fígado, coração, dentre outros, e Soares et al., 1999 registraram formas de $P$. juxtanucleare em leucócitos e trombócitos de $G$. gallus.

Com o objetivo de ampliar o conhecimento sobre a ocorrência e as relações do parasitismo de $P$. juxtanucleare em $G$. gallus, foi feito um levantamento desse parasito, com ênfase na intensidade de infecção, no município de Santa Bárbara do Tugúrio, estado de Minas Gerais.

\section{Material e métodos}

As aves estudadas foram alimentadas com milho, frutas e pastagem, mantidas soltas em locais sem cuidados com higiene, não havendo medidas profiláticas contra doenças aviárias.

Foram analisadas amostras sangüineas de 30 aves mestiças, adultas, sendo 29 fêmeas e um macho, de criação rústica, mantidas extensivamente, com peso médio de $2,18 \mathrm{~kg} \pm$ 0,34 , provenientes do sítio Boa Vista, no município de Santa Bárbara do Tugúrio (2115'12"S e 4327'16"W), Campo das Vertentes, Minas Gerais, Brasil.

Dados de identificação de cada hospedeiro, como aspecto físico, comportamento, manejo, idade, sexo, peso, data da coleta e o resultado do exame dos esfregaços foram registrados.

As amostras de sangue foram obtidas por venopunção radial, sendo confeccionados esfregaços finos, secados ao ar. As lâminas foram identificadas na sua porção mais espessa, indicando o número da ave. Os esfregaços foram fixados em metanol e corados pelo Giemsa.

As formas eritrocitárias foram registradas e quantificadas pela observação de 50 campos em microscopia fotônica em imersão, assinalando-se o número de eritrócitos parasitados.

\section{Resultados e discussão}

Ao exame dos esfregaços sangüíneos, todas as aves mostraram-se positivas à infecção de $P$. juxtanucleare, correspondendo a uma prevalência de $100 \%$. Os dados de prevalência obtidos foram superiores àqueles observados por diversos autores, como Ferraz Franco et al. (1954) 7,3\% e Krettli (1971) 19\% no estado de Minas Gerais; Massard (1979) $58,1 \%$ em municípios do estado do Rio de Janeiro, Minas Gerais e Espírito Santo; Massard e Massard (1981) 38,7\% no estado do Rio de Janeiro; Mota (1997) $28,4 \%$ na região metropolitana do Recife e Souza (1998), 35,5\% no estado do Rio de Janeiro.
Pode-se verificar uma intensidade média de 7,93 parasitos por lâmina, observando-se em 33,3\% dos esfregaços de um a cinco parasitos, $40 \%$ de seis a dez e $26,6 \%$ acima de dez. Os resultados dos exames sangüíneos demonstrando a intensidade de infecção estão expostos na Figura 1.

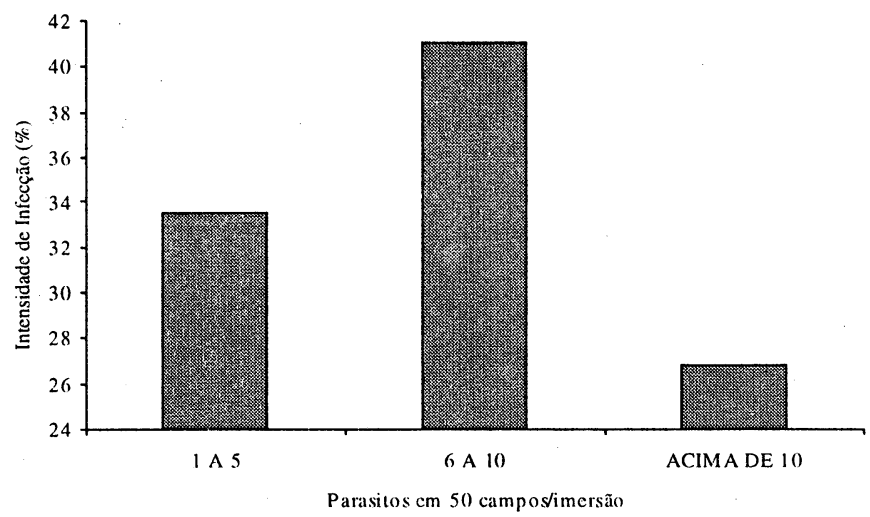

Figura 1: Intensidade média de infecção de Plasmodium juxtanucleare por esfregaços sangüíneos de Gallus gallus L., de criações rústicas no município de Santa Bárbara do Tugúrio, MG

Dentre os trabalhos que estimaram a intensidade de infecção por P. juxtanucleare, Kretlli (1971), Massard (1982), Mota (1997) e Souza (1998), estipularam a avaliação de 100 campos microscópicos para a determinação do número de eritrócitos parasitados ou para se considerar a negatividade do esfregaço. Para um melhor estudo desta infecção e a padronização da parasitemia para fins de diagnóstico, Souza et al. (1999) propuseram a contagem de hemácias em 20 campos homogêneos no terço final do esfregaço, em $5 \%$ do total de lâminas a serem examinadas, calcular a média do número de eritrócitos/campo e através de regra de três simples, calcular o número de campos microscópicos a serem pesquisados para atingir o total de 10 mil hemácias. No presente trabalho, os 50 campos selecionados mostram-se suficientes, uma vez que a infecção foi constatada em todos os esfregaços, apesar do baixo número de parasitos por campo.

Não se verificou a presença de eritrócitos com poliparasitismo e, concordando com Mota et al. (1998), não se observaram aspectos ou comportamentos sugestivos da malária aviária, indicativo de uma infecção crônica e assintomática. No entanto, é uma infecção responsável por altas taxas de morbidade e mortalidade. Em criações industriais ela está relacionada com a queda na produção de ovos, como citado por Massard (1982). Soares et al. (1999) mencionaram um quadro de anemia, febre, inapetência, distúrbios digestivos, distúrbios neurológicos e megalia dos órgãos.

A elevada prevalência de $100 \%$ de infecção por $P$. juxtanucleare registrada no presente trabalho, indica que o local apresentava, no momento do estudo, condições ideais para a transmissão e manutenção do parasito em galinhas e mosquitos hospedeiros invertebrados. Estudos complementares devem ser realizados no local para o conhecimento das condições ambientais que favoreceram tal prevalência e da estabilidade, ou não, desse índice no local e na região. 


\section{Referências}

FERRAZFRANCO, H., VAITSMÁN, J. e MOUSSATCHÉ, J. Hemoparasito em aves domésticas. Rev. Mil. Rem. Vet. v.14, p. 29-3, 1954.

GARNHAM, P. C. C. Malaria Parasites and Other Haemosporidia. Blackwell Sci. Public. Oxford, 1.114 p, 1966.

KRETTLI, A.U. Estudos sobre a prevalência, biologia e transmissão do Plasmodium juxtanucleare Versiani e Gomes, 1941. 1971.73 p. Dissertação (Mestrado) - Universidade Federal Rural do Rio de Janeiro, Seropédica, RJ.

MASSARD, C. L. Aspectos biológicos de Plasmodium juxtanucleare Versiani e Gomes, 1941 em aves do Brasil. 1976. 53 p. Dissertação (Mestrado). Universidade Federal Rural do Rio de Janeiro, Seropédica, RJ.

MASSARD, C. L. Significância das infecções causadas porPlasmodium (Noyella) juxtanucleare Versiani e Gomes, 1941 (Haemosporida: Plasmodiidae) em Gallus gallus $L$. de criação industrial no estado do Rio de Janeiro. 1979.57p. Tese (Doutorado) - Universidade Federal Rural do Rio de Janeiro, Seropédica, RJ.

MASSARD, C. L. Caracterização do parasitismo por Plasmodium juxtanucleare (Haemosporidea: Plasmodiidae) em criação de Gallus gallus da raça Leghorn Branca. Arq. Univ. Fed. Rur. Rio de J., v. 5, p.141-146, 1982.

MASSARD, C.L e MASSARD, C. A. Aspectos biológicos do Plasmodium juxtanucleare Versiani e Gomes, 1941 em aves do Brasil. Rev.Bras.Med.Vet., v. 4, p. 3-24, 1981.

MOTA, R.A. Variações hematológicas em Gallus gallus domesticus Linnaeus, 1758 , de criações rústicas da região metropolitana do Recife, naturalmente infectados com o Plasmodium (Novyella) juxtanucleare Versiani e Gomes, 1941. 1997, 58 p. Tese (Doutorado) - Universidade Federal Rural do Rio de Janeiro, Seropédica, RJ.

MOTA, R.A., CUNHA, E.L.P., SOARES, C.O., ALVES, L.C. e MASSARD, C. L. Variáveis Hematológicas em Gallus gallus domesticus, Linneaus, 1758, de criações rústicas da região metropolitana do Recife, natural- mente infectados com Plasmodium juxtanucleare(Versiani e Gomes, 1941). Ciênc. Vet. Trop. Recife, v.1, p. 76-80, 1998.

PARAENSE, W. L. Um inquérito sobre a ocorrência de Plasmodium juxtanucleareem Bambui (estado de Minas Gerais). Mem. Inst. Oswaldo Cruz, v. 47, p. 361-365, 1949.

SERRA FREIRE, N.M e MASSARD, C. L. Ocorrência e incidência de Plasmodium juxtanucleare Versiani e Gomes, 1941, em Belém-Pará. CONGRESSOSOCIEDADEBRASILEIRAPARASTTOLOGIA, 1976, Belém, Anais ... SBP.

Plasmodium juxtanucleare Versiani e Gomes, 1941, parasita de

Gallus gallusL., Meleagris gallopavo L.e Crysolophusspp na região do Pantanal do estado do Mato Grosso do Sul. Atas. Soc. Biol. Rio de Janeiro,v. 20, p. 45-48, 1979.

SOARES, C. O., SOUZA, P. C. A., MOTA, R. A., FONSECA, A. H. e MASSARD C. L. Parasitismo de leucócitos e trombócitos de Gallus gallus L. por Plasmodium (Novyella) juxtanucleare (Apicomplexa: Plasmodiidae). Parasitol. al Dia. v. 23, p. 44-47, 1999.

SOUZA, P. C. A. Malária Aviária: Parasitismo por Plasmodium (Novyella) juxtanucleare Versiani e Gomes, $1941 \mathrm{emGallus} \mathrm{gallus} \mathrm{L.}$ de criações rústicas, nas mesorregiões do estado do Rio de Janeiro e aspectos clínicos e patológicos de sua infecção experimental. 1998. 137 p. Tese (Doutorado) - Universidade Rural do Rio de Janeiro, Seropédica, RJ.

SOUZA, P. C. A., SOARES, C. O., FREIXO, A. P. A., BERNER, P. G. M.; MASSARD, C. L. e LOPES, C. W. G. Proposta para padronização do cálculo da parasitemia em infecções pelo Plasmodium juxtanucleare. SEMINÁRIO BRASILEIRO DE PARASITOLOGIA VETERINÁRIA. ResUmo. Salvador, BA. 1999. p. 213.

VERSIANI, V. e GOMES, B. F. Sobre um novo hematozoário de galinha, Plasmodium juxtanucleare n. sp. (nota prévia). Rev. Bras. Biol., v.1, p. 231-233, 1941.

VERSIANI, V. e GOMES, B. F. Plasmodium juxtanucleare parasita de galinha doméstica (notas adicionais). Rev. Bras. Biol., v., p. 113-117, 1943. 